\title{
SKA studies of nearby galaxies: star-formation, accretion processes and molecular gas across all environments
}

\author{
R. J. Beswick ${ }^{* 1}$ E. Brinks ${ }^{2}$, M. A. Pérez-Torres ${ }^{3}$, A. M. S. Richards ${ }^{1}$, S. Aalto ${ }^{4}$, A. \\ Alberdi $^{2}$, M. K. Argo ${ }^{1}$, I. van Bemmel ${ }^{5}$, J. E. Conway ${ }^{4}$, C. Dickinson ${ }^{1}$ D. M. Fenech ${ }^{6}$, \\ M. D. Gray ${ }^{1}$, H-R Klöckner ${ }^{7}$, E. J. Murphy ${ }^{8}$, T. W. B. Muxlow ${ }^{1}$, M. Peel ${ }^{1}$, A. P. \\ Rushton $^{9,10}$, E. Schinnerer ${ }^{11}$ \\ ${ }^{1}$ Jodrell Bank Centre for Astrophysics/e-MERLIN, The University of Manchester, M13 9PL, UK \\ ${ }^{2}$ Centre for Astrophysics Research, University of Hertfordshire, AL10 9AB, UK \\ ${ }^{3}$ Instituto de Astrofisica de Andalucia (IAA-CSIC), E-18008 Granada, Spain \\ ${ }^{4}$ Department of Eath and Space Sciences, Chalmers University of Technology, Onsala Space \\ Observatory, SE-439 92, Onsala, Sweden \\ ${ }^{5}$ Netherlands Institute for Radio Astronomy (ASTRON), Postbus-2, NL-7990 AA Dwingeloo, The \\ Netherlands \\ ${ }^{6}$ Department of Physics and Astronomy, University College London, London, WC1E 6BT, UK \\ ${ }^{7}$ Max-Planck-Institut für Radioasronomie, Auf dem Hügel 69, D-53121 Bonn, Germany \\ ${ }^{8}$ IPAC, Caltech, MC 220-6, Pasadena CA, 91125, USA \\ ${ }^{9}$ Department of Physics, Astrophysics, University of Oxford, Keble Road, Oxford, OX1 3RH, UK \\ ${ }^{10}$ School of Astronomy and Physics, Univeristy of Southampton, highfield, Southampton, \\ SO17 1BJ, UK \\ ${ }^{11}$ Max-Planck-Institut für Astronomie, Königstuhl 17, D-69117, Heidelberg, Germany
}

\begin{abstract}
The SKA will be a transformational instrument in the study of our local Universe. In particular, by virtue of its high sensitivity (both to point sources and diffuse low surface brightness emission), angular resolution and the frequency ranges covered, the SKA will undertake a very wide range of astrophysical research in the field of nearby galaxies. By surveying vast numbers of nearby galaxies of all types with $\mu \mathrm{Jy}$ sensitivity and sub-arcsecond angular resolutions at radio wavelengths, the SKA will provide the cornerstone of our understanding of star-formation and accretion activity in the local Universe. In this chapter we outline the key continuum and molecular line science areas where the SKA, both during phase-1 and when it becomes the full SKA, will have a significant scientific impact.
\end{abstract}

Advancing Astrophysics with the Square Kilometre Array

June 8-13, 2014

Giardini Naxos, Italy

\footnotetext{
* Speaker.

†Robert.Beswick@manchester.ac.uk
} 


\section{Introduction}

The appearance of galaxies, and by this virtue, our Universe as a whole is dominated by two physical processes: star-formation and accretion. Star-formation (SF) is fundamental to the formation and evolution of galaxies, whilst accretion provides a major power source in the Universe, dominating the emission from distant quasars down to nearby X-ray binary systems. The feedback between these two processes is also crucial, e.g., in reconciling the observed galaxy luminosity function with predictions from the standard hierarchical clustering models. Radio observations provide by far the best single diagnostic of these two processes, allowing a direct view of SF even in dusty environments and the detection of AGN and measurement of their accretion rate at bolometric luminosities far below anything detectable at higher energies.

In this chapter we outline the scientific motivation for undertaking large surveys and deep observations of galaxies in the local Universe across all available frequency bands of SKA1 and look forward to the full SKA. The high sensitivity, resolution and imaging fidelity capabilities of SKA1, and the expected enhanced capabilities of the full SKA, will ensure that the SKA will become a dominant instrument for the detailed study of nearby galaxies over the coming decades.

Continuum surveys, undertaken during phase-1 covering the expected SKA dish (MID/SUR) frequency ranges of $0.35-3 \mathrm{GHz}$ and higher (see also Murphy et al., 2015, in this proceedings), will provide $\mu \mathrm{Jy}$ sensitivities with exquisite image fidelity over a wide range of spatial scales for all nearby galaxies. This will produce a complete census of SF and AGN activity as a function of galaxy mass, morphology and spectral type, black-hole mass and luminosity; alongside opti$\mathrm{cal} / \mathrm{IR} / \mathrm{mm}$ surveys and observations from ALMA, LSST, VISTA, Spitzer, Herschel and WISE, this will be the cornerstone of multi-wavelength studies of the local Universe.

By providing high angular resolution observations ( $\sim 0^{\prime \prime} 5$ or better) the SKA will be able to decompose individual galaxies into their compact radio source populations comprising accretion dominated AGN plus the tracers of the early stages of SF, such as compact HII regions, super star clusters (SSCs), as well as stellar end-points like X-ray binaries, planetary nebulae, supernovae (SNe) and their remnants (SNR). In nearby galaxies the SKA will image these sources at physically important size scales ( $\sim$ few to tens of parsecs) characterising individual sources and providing a detailed extinction-free census of the compact SF products across a wide range of galaxy types and the environment parameter space they inhabit. For example, at a sensitivity of a few $\mu \mathrm{Jy}$ at $\sim \mathrm{GHz}$ frequencies, a census of this type will detect all of the long-lived radio SNR within several tens of $\mathrm{Mpc}$, thus providing a measure of SF rates (SFRs) within local galaxies, independent of the IRradio correlation and obscuration corrections. The mismatch between the measured Core Collapse SN (CCSNe) rates and the cosmic massive SFR (Mattila et al., 2012) should be corrected. Such a measure will preferentially trace high mass $\mathrm{SF}\left(\mathrm{M}>8 \mathrm{M}_{\odot}\right)$, providing a direct tracer of the upper part of the galaxy Initial Mass Function (IMF). The SKA will detect all AGN activity in the local Universe. By resolving and measuring the AGN and SF contributions, local Universe studies of galaxies with the SKA will be vital in determining the contributions and interplay of these two key physical processes which are critical for our interpretation of the high redshift Universe. On larger scales the SKA will image the diffuse radio emission structure of nearby galaxies across a range of frequencies (few hundred $\mathrm{MHz}$ - several $\mathrm{GHz}$, ideally up to at least $13 \mathrm{GHz}$ ) thus allowing the separation of synchrotron and thermal radio emission. The component separation of the thermal 

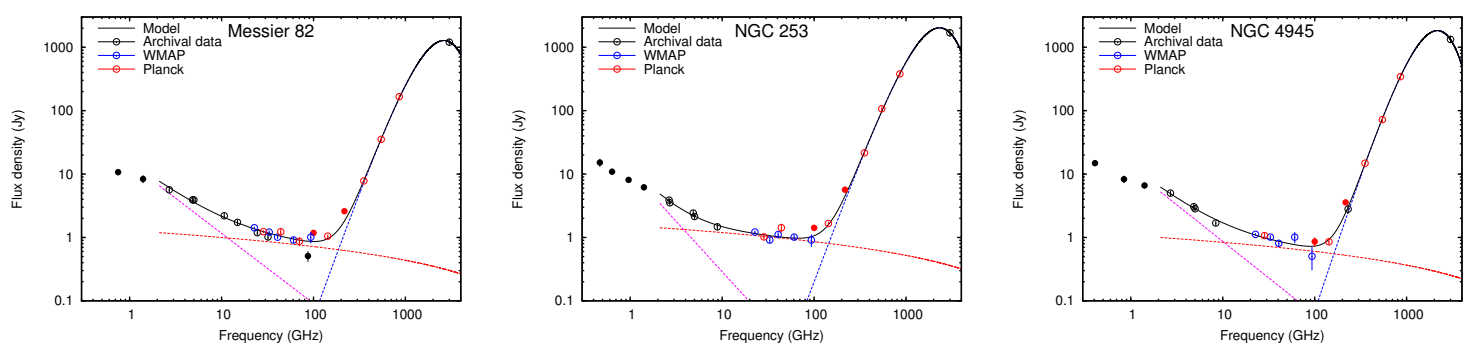

Figure 1: Spectral energy distribution of the nearby star-forming galaxies M82, NGC253 and NGC4945. Spectral fits to synchrotron (magenta), thermal (free-free - red) and thermal (dust - blue) are shown alongside the combination of these products as a solid line (Peel et al., 2011).

emission of galaxies provides another unobscured measure of the massive SF activity spanning a diverse range of galaxy types and environments, and providing a local Universe reference point.

Observations of this type will be undertaken both commensally with other wide-area or pointed observations (Prandoni \& Seymour, 2015), along with specific targeted pointings focusing on this science theme. Complementing the continuum science for nearby galaxies, high sensitivity cold neutral, molecular and ionised ISM observations (the fuel for the SF and accretion processes traced via continuum observations, see section 4, also see de Blok et al. 2015; Paladino et al. 2015; Oonk et al. 2015 - this proceedings), and polarization observations of galaxies (Beck et al., 2015; Heald et al., 2015, also in these proceedings) will also be made by the SKA. This combined information will allow the SKA to fully characterise the ongoing physics and fueling of SF and accretion within the nearby galaxy population.

\section{Radio continuum tracers of star-formation in the local Universe}

The SF history, along with current levels of SF, within individual galaxies still remains a crucial physical parameter that observations are only now beginning to accurately characterise. Traditionally, observations of optical line, IR and the global synchrotron emission from galaxies have been used as proxies for SF. However, these tracers have some fundamental flaws. In many galaxies optical emission lines are heavily obscured towards their centres, this can require potentially large corrections, whilst IR emission, which essentially traces the light from young stars reprocessed by dust and re-radiated at longer wavelengths, relies upon empirical interpretation of physically complex processes in order for it to be related to SF. Global radio synchrotron emission provides an alternative, and extinction-free, indication of SF, however, the link between radio synchrotron emission and SF is also via complex physical mechanisms (see section 2.1.1) which are generally calibrated using the radio-to-IR correlation (Yun, Reddy \& Condon, 2001; Bell, 2003; Beswick et al., 2008). The long-standing critical issue which remains is how to calibrate either global radio or IR emission as a measure of SF, and how this calibration varies as a function of galaxy properties (e.g. gas content, SFR, specific SFR, interaction/merger state) and their global environment (Kennicutt, 1998). 


\subsection{Thermal and non-thermal radio emission as star-formation tracers}

\subsubsection{Radio-Infrared correlation and star-formation}

The physical underpinning of the radio-continuum (RC) - Far-Infrared (FIR) correlation is broadly understood to be the formation of massive stars, which can believably be associated with the dominant contributions to both the FIR and RC emission of galaxies. The IR contribution is due to the hot dust envelopes surrounding the massive stars, which are energised by the copious amounts of Lyman continuum radiation that those stars emit. The radio contribution includes two components (see Fig. 1). The first is the thermal continuum, free-free emission, which predominantly comes from Hil regions, associated with massive $\left(M \geq 8 \mathrm{M}_{\odot}\right)$ stars. The second is the non-thermal, synchrotron emission from relativistic electrons. These electrons are accelerated in the SNe shocks that occur when massive stars explode as CCSNe (type Ibc and type II SNe). When the supernova blast wave interacts with the outer material a shell is formed, and radio emission is generated initially due to the interaction with the circumstellar medium (CSM), fading away with time as the supernova continues to expand. Eventually, the supernova shock meets the uniform interstellar medium (ISM), at which time the SN suffers a re-brightening and continues emitting at radio wavelengths for thousands of years, now as a SNR, which is also a very efficient cosmic ray electron (CRe) accelerator. If all CRe lose their energy exclusively within the galaxy, it can be considered a calorimeter (Völk, 1989; Lisenfeld, Völk \& Xu, 1996).

Both continuum thermal free-free radio emission and synchrotron radio emission can be considered as SF tracers. Thus if the presence of an AGN can be excluded (or its contribution neglected), one could then obtain independent estimates of the SF rate, and thus check the linearity of the RC(thermal)-FIR and RC(sync)-FIR correlations.

RC and FIR emission both depend on (recent) SF and will thus be correlated. Surprisingly, this RC-FIR relation of galaxies holds over 4 orders of magnitude in luminosity, irrespective of galaxy type (Helou et al., 1985; de Jong et al., 1985; Beswick et al., 2008); displays only a 0.26 dex scatter, and has been observed to hold out to a redshift of about 3 (Garrett, 2002; Appleton et al., 2004). Fig. 2 (left hand panel) taken from Kitchener et al. (2014), which is based on data from Yun, Reddy \& Condon (2001), shows the RC-FIR relation based on the integrated emission from star forming (spiral) galaxies. The RC-FIR relation can be understood if not only the CRe lose all their energy within the galaxy but also the dust absorbs and transforms all the emission related to recent SF into FIR. More realistically, a galaxy is a leaky box, and models become far more involved (e.g., Bell, 2003; Beswick et al., 2008; Lacki, Thompson \& Quataert, 2010). Despite the leaky nature of galaxies, the relative successes of theoretical models lend confidence that massive SF and RC emission are indeed closely tied together. A well calibrated RC-FIR relation offers a powerful method to probe the cosmic SFR out to intermediate redshifts, initially with SKA pathfinders and precursors, and eventually with the SKA (e.g., Murphy, 2009; Lacki, Thompson \& Quataert, 2010; Murphy et al., 2012; Norris et al., 2013). This will be an essential prerequisite for deep extragalactic surveys with the SKA since measuring the cosmic SF history will be limited by our understanding of the radio continuum-to-SF relationship rather than the number count statistics which limit today's observations.

Rather than relying on the FIR, which at best provides modest angular resolution and relies on the availability of suitable satellites, one can use the RC to determine directly the current SFR 

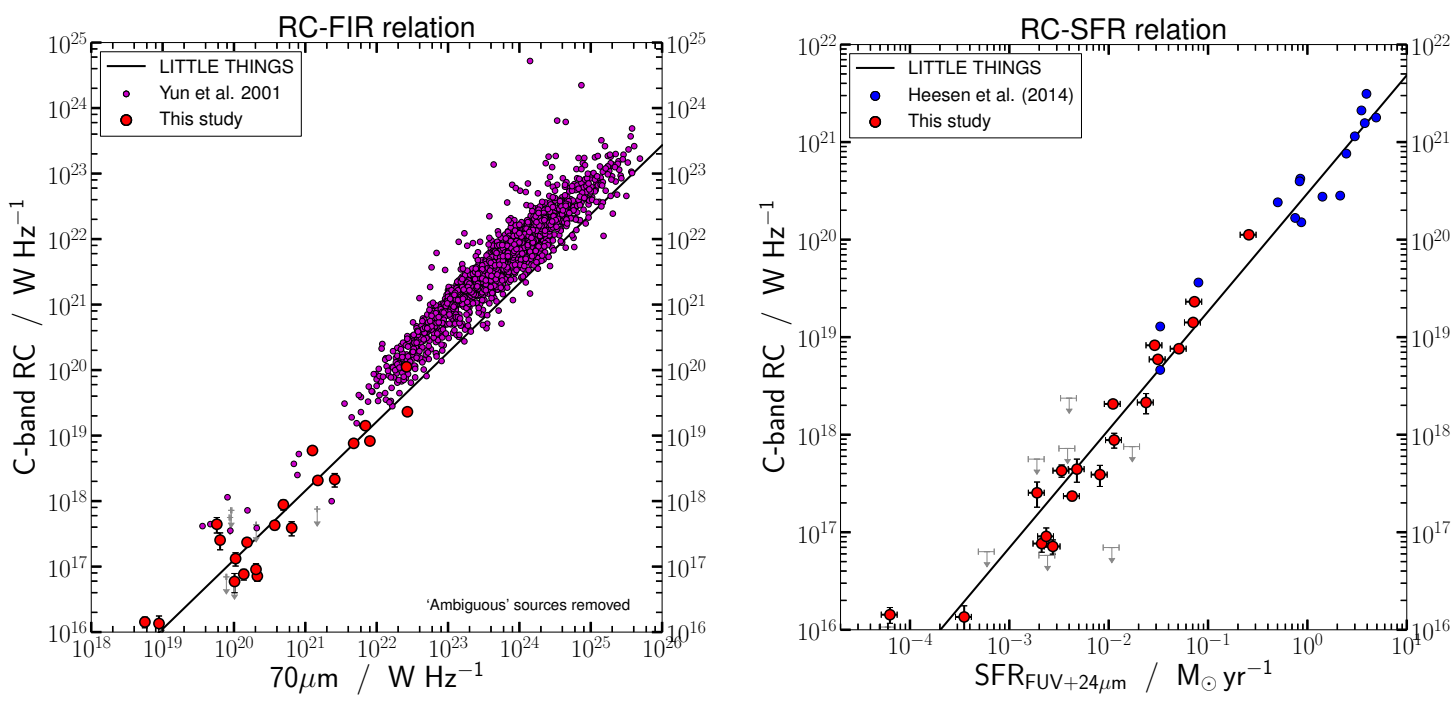

Figure 2: left: $R C$-FIR relation Filled purple symbols are integrated values for the galaxies taken from the sample collected by Yun, Reddy \& Condon (2001). Their VLA 1.4 GHz data have been corrected to what would be expected at $6 \mathrm{GHz}$ and their IRAS $60 \mu \mathrm{m}$ data to the Spitzer $70 \mu \mathrm{m}$ passband. The red circles and upper limits (downward arrows) come from the $6 \mathrm{~cm}$ VLA survey of 40 dwarf irregular galaxies by Kitchener et al. (2014) and are listed as "This study" in the legend. The line is a fit through the red circles and corresponds to a power law slope of $0.9 \pm 0.1$ and a dispersion of 0.3 dex. right: $R C-S F R$ relation The blue circles are from Heesen et al. (2014) who mainly studied spiral galaxies observed with the WSRT at $22 \mathrm{~cm}$ from the SINGS survey. The WSRT $22 \mathrm{~cm}$ fluxes have been corrected to $6 \mathrm{GHz}$. The red circles and upper limits (downward arrows) are from Kitchener et al. (2014).

in galaxies (see Condon, 1992, for a review). The thermal and non-thermal emission are the result of fundamentally different processes, with different RC-SFR relations expected for each component. The thermal RC is expected to be directly proportional to the SFR, depending as it does on the ionised flux from massive stars. This makes it an ideal, virtually extinction-free proxy for SF (Murphy et al., 2011). The non-thermal RC depends on the magnetic field strength as well as the cosmic-ray energy density, unless one assumes an electron calorimeter which is unlikely, particularly for dwarf galaxies, or in general those galaxies with large-scale outflows. Usually one assumes energy equipartition between the CRe and the magnetic field, so that the RC-SFR relation is closely connected to a relation between the magnetic field and gas. Price \& Duric (1992) separated the RC into its thermal and non-thermal components and found both to follow their own unique RC-FIR relation; the thermal relation being indistinguishable from a one-to-one relation (power law slope of $0.97 \pm 0.02$ ) whereas the non-thermal relation is steeper, at $1.33 \pm 0.10$. Although it is less obvious why the non-thermal RC would form a tight relation with SF, empirically it does and recent theoretical work suggests the non-thermal RC-SFR follows a super-linear (e.g., Niklas \& Beck, 1997; Schleicher \& Beck, 2013) relation with a slope of RC $\sim$ SFR ${ }^{1.3}$. Given that, at the lower frequencies, non-thermal emission related to SF can be 1-2 orders of magnitude brighter than the thermal counterpart, there is great potential in arriving at a well calibrated and understood RC-SFR relation. A recent compilation is shown in Fig. 2 (right hand panel) taken from Kitchener et al. (2014) which is based on data from Heesen et al. (2014). It shows the RC-SFR relation based on the integrated emission from star forming galaxies. 

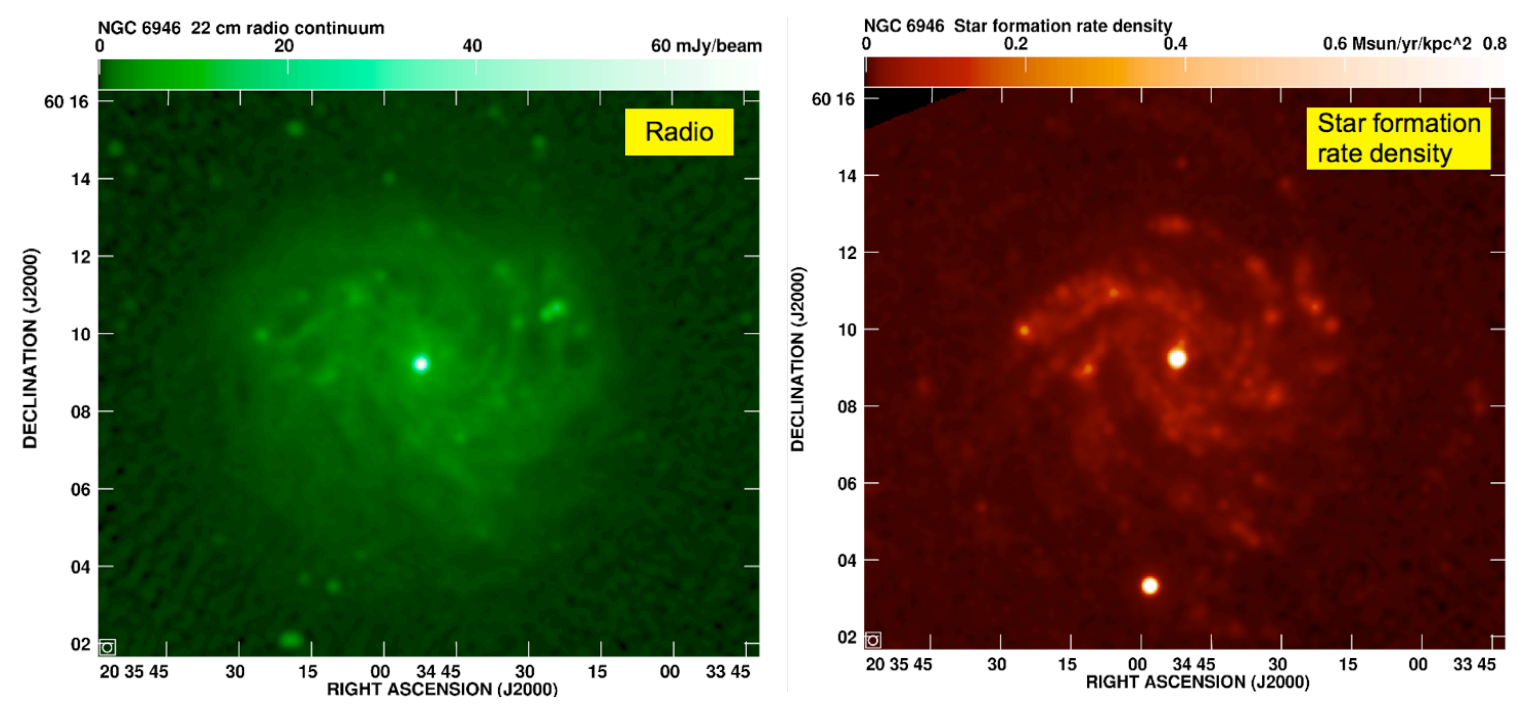

Figure 3: Images of the $22 \mathrm{~cm}$ radio continuum and derived star-formation rate density for the nearby face-on starforming galaxy NGC 6946.

\subsubsection{From existing capabilities to the SKA}

Upgrades to existing instruments (in the form of wide-band end-to-end systems) have opened up a number of possibilities including i) spatially resolved imaging of dozens of spirals in the Local Universe down to $\mu \mathrm{Jy}$ levels, ii) extending those studies to the low mass, low metallicity regime of dwarf irregular galaxies, and iii) mapping of nearby galaxies at parsec resolution which, via a headcount of individual SNRs, gives an independent handle on the SFR (Fenech et al., 2008, 2010). Murphy et al. $(2006,2008)$ provide spatially resolved information at $\sim 1 \mathrm{kpc}$ scale, using Westerbork Synthesis Radio Telescope (WSRT) $20 \mathrm{~cm}$ observations (Braun et al., 2007) combined with Spitzer $70 \mu \mathrm{m}$ maps. They find that the RC emission mimics a smoothed version of the FIR map, a consequence of CR electrons diffusing over their $\sim 10^{8}$ yr lifetime across typically $\sim 1 \mathrm{kpc}$. They also find that the slope of the RC-FIR relation, $q_{70}$, varies with radius and with surface brightness. Fig. 3 shows the stunning similarity between $22 \mathrm{~cm}$ radio continuum emission from Braun et al. (2007) and a map tracing the current SFR which is based on a linear combination of GALEX FUV and Spitzer $24 \mu \mathrm{m}$ emission (based on Heesen et al., 2014). These authors, based on a sample of 18 nearby spirals, conclude that the RC-SFR relation as posited by Condon (1992) holds for their integrated luminosities. When averaging azimuthally, the ratio of $22 \mathrm{~cm}$ surface brightness to the SFR surface density is remarkably constant as well. Diffusion of CRe flattens the local (averaged over a 0.7 to $1.2 \mathrm{kpc}$ diameter area) RC-SFR relation, and this is compatible with magnetic field amplification by a small-scale dynamo, powered by SF-driven turbulence.

Kitchener et al. (2014) push these results to the low-mass, low SFR regime of dwarf irregulars (the red symbols in Fig. 2). They find that dwarf galaxies follow the same RC-FIR slope as that defined by the larger spirals, but are systematically fainter by a factor of about two. Assuming equipartition, the strength of the B-field is $9 \mu \mathrm{G}$, which is of the same order as that in spirals. Resolved studies in nearby spiral galaxies such as M51 (Dumas et al., 2011) and NGC6946 
(Tabatabaei et al., 2013) show deviations in the RC-IR slope as a function of environment (e.g. arm vs. inter-arm, SFR, gas density and radiation field). These studies find evidence of clear variations of the synchrotron emission with SFR thus providing further evidence for coupling of the gas density and magnetic field strength. At SFRs lower than $1 \mathrm{M}_{\odot} \mathrm{yr}^{-1}$, the RC deviates increasingly from the Condon relation, which is interpreted as being due to a deficiency of non-thermal emission as a result of CRe escape.

SKA1-MID will transform what can be achieved. Rather than monochromatic (or at best inband spectral index) information, observations of about $1 \mathrm{hr}$ per band will sample the entire radio continuum spectrum between 1.67 and $10 \mathrm{GHz}$ (about $4 \mathrm{hr}$ total per target), down to $\mu$ Jy sensitivity. This will allow the above mentioned relations to be put on a much more robust footing as we will be able to separate off the thermal contribution, leaving just the non-thermal fraction. In addition to extending the work quoted above by Murphy et al. (2011), Heesen et al. (2014) and Kitchener et al. (2014) to larger samples, the spatially resolved spectral index distribution will come within reach, which should provide a lever on the propagation and aging of CRe as they diffuse from their sites of origin.

\subsection{Decomposition of local galaxies into their constituent parts}

Sensitive and critically high angular resolution $(\leq 0$." 5$)$ radio images of nearby galaxies such as will be provided by the SKA1-MID and by the complete SKA, provide one method by which observations can directly probe SF and SF processes in a way which is independent of complex physical emission mechanisms. Whereas lower resolution radio observations of normal and starforming galaxies trace the diffuse radio emission (see Section 2.1.1), sub-arcsecond angular resolution observations are required to systematically characterise the populations of individual compact SF products on a galaxy by galaxy basis by resolving away the diffuse emission. This population census can hence be used to directly infer the levels of SF.

Observed with $<0$." 5 angular resolution, each individual galaxy can be considered as a laboratory containing a large sample of discrete radio sources, all at essentially the same distance, which can be studied in a systematic way. At $\mu \mathrm{Jy}$ and sub- $\mu \mathrm{Jy}$ sensitivities and between frequencies of 1 and $7 \mathrm{GHz}$ this source population, with the exception of accretion dominated objects and in particular AGN (see Section 3), will consist exclusively of sources related to various key phases of the stellar evolutionary sequence. This population will be a mixture of sources from the early stages of SF, such as compact HII regions, through SSCs, and stellar end-points like X-ray binaries, planetary nebulae, $\mathrm{SNe}$ (see also Pérez-Torres et al., 2015, in this proceedings) and their SNR.

By first detecting and then identifying the physical nature of these objects using a combination of radio morphologies and spectral indices, alongside extensive multi-wavelength ancillary data, high angular resolution radio observations will provide the first detailed extinction-free census of $\mathrm{SF}$ products within nearby galaxies. The majority of core-collapse SNe evolve to form long-lived radio SNR (Weiler et al., 2002; Gendre et al., 2013), hence this statistically well-constrained census combined with information regarding the sizes and hence canonical ages of SNR (for the nearest galaxies observed with sub-arcsecond resolution available in higher bands with SKA1-MID), can be used to directly infer levels of SF in individual galaxies (e.g. Pedlar, 2001; Fenech et al., 2008, 2010). This will provide a further obscuration-independent SFR tracer and, because it detects massive stars which form CCSNe, will preferentially probe the top part of the IMF. When combined 
with other SFR measures such as IR/UV or global RC free-free and synchrotron emission (e.g. Section 2.1.1) this will provide constraints on the universality of the IMF as a function of galaxy type, evolution and environment within the local volume.

Importantly, such a local galaxy radio survey will also identify populations of sources that trace earlier stages in stellar evolution, such as HII regions and SSCs, placing useful constraints on the levels of SF at various phases in the evolution of individual galaxies. When compared with other wavelength tracers, which probe different ranges of SF age and different spatial regions, these radio diagnostics will provide significant new insights.

Whilst this will be achievable on a galaxy-by-galaxy basis, the power and importance of an SKA survey of nearby galaxies arises from its large size and the available complementary multiwavelength data-sets. By combining these direct radio tracers of SF products, with other multiwavelength SF proxies (e.g. IR), significant constraints will be placed on their calibration and interpretation, with important implications across a wide range of observational astrophysics. Largearea SKA1 continuum surveys with both SKA-MID and SKA-SUR with sensitivities of $\sim 4 \mu \mathrm{Jy}$ will be capable of detecting all local galaxies thus spanning a complete range of types and levels of both historical and ongoing SF, and allowing this census of SF products to be applied over the wide range of luminosity and environment parameter space inhabited by galaxies. Such a survey will provide the radio benchmark for studies of local galaxies with application to all observational astronomers.

\subsection{Towards higher redshift}

Intermediate redshift $(0.1<\mathrm{z}<0.3)$ and some local Luminous Infrared Galaxies (LIRGs) and Ultra-Luminous Infrared Galaxies (ULIRGs) are thought to be nearby versions of high-z starforming galaxies (see also Mancuso et al., 2015, in this proceedings). Since a major science goal for the SKA and its pathfinders is the study of SF across cosmic time, it is crucial to i) have a detailed and accurate knowledge of local star-forming galaxies, and ii) test the radio-infrared (radio-IR) relation. Since radio emission is a dust-unbiased SF tracer, an accurate calibration of the radio-IR relation will be needed to determine the SFRs at high-z. Indeed, at $z \geq 1,1 \operatorname{arcsec}$ corresponds to $8 \mathrm{kpc}$, so that disentangling AGN from star-forming activity is challenging, unless angular resolutions better than $\sim 0$.' 1 are provided, so that one starts to separate a putative AGN from a compact starburst at essentially any redshift. This capability will be provided by the full SKA.

A large fraction of massive SF at both low- and high- $z$ has taken place in (U)LIRGs. Their implied high SFRs are expected to result in CCSN rates a couple of orders of magnitude higher than in normal galaxies. Therefore, a powerful tracer for starburst activity in (U)LIRGs is the detection of CCSNe, since the SFR is directly related to the CCSN rate. However, most SNe occurring in ULIRGs are optically obscured by large amounts of dust in the nuclear starburst environment, and have therefore remained undiscovered by (optical) SN searches. Fortunately, it is possible to discover these CCSNe through high-resolution radio observations, as radio emission is free from extinction effects. Furthermore, $\mathrm{CCSNe}$ are expected (unlike thermonuclear $\mathrm{SNe}$ ) to become strong radio emitters when the SN ejecta interact with the CSM that was ejected by the progenitor star before its explosion as a supernova. Therefore, if (U)LIRGs are starburst-dominated, bright radio $\mathrm{SNe}$ are expected to occur. Given their compactness and characteristic radio behavior of radio $\mathrm{SNe}$ they can be pinpointed with high-resolution, high-sensitivity radio observations (e.g., SN $2000 \mathrm{ft}$ in 
NGC 7469 Colina et al. 2001; Alberdi et al. 2006; Pérez-Torres et al. 2009b; SN 2004ip in IRAS 18293-3413, Pérez-Torres et al. 2007; SN 2008cs in IRAS 17138-1017, Pérez-Torres et al. 2008; Kankare et al. 2008; supernovae in Arp 299 Neff, Ulvestad \& Teng 2004, Arp 220 Smith et al. 1998; Lonsdale et al. 2006; Parra et al. 2007, Mrk 273 Bondi et al. 2005). However, since (U)LIRGs are likely to have an AGN contribution, high-sensitivity, high-resolution radio observations are required to disentangle the nuclear and stellar (mainly from young $\mathrm{SNe}$ ) contributions to the radio emission, thus probing the mechanisms responsible for the heating of the dust in their (circum)nuclear regions.

In view of the importance of (U)LIRGs in tracing the SF history across cosmic time, a targeted survey of local (U)LIRGs using SKA1-MID, up to a distance of $100 \mathrm{Mpc}$, will be essential. The sub-arcsecond angular resolution in band $2 / 3$ will be well-matched to current (J)VLA-A images at higher frequencies, permitting the thermal and non-thermal contribution to be disentangled in the very centres of galaxies. Considering the continuum sensitivity provided by SKA1-MID, as little as 2 minutes per source would be sufficient to produce $1.7 \mathrm{GHz}$ images of a similar depth to those currently provided by the JVLA at $8.4 \mathrm{GHz}$ in 1 hour. A census of all local (U)LIRGs could be obtained in just a few hours with SKA1-MID. Even if the specifications deviate by as much as $30 \%$ in terms of sensitivity, this science will not be severely affected. However, baseline lengths of at least $200 \mathrm{~km}$ are required, to provide the necessary angular resolution. An SKA-MID survey providing an angular resolution of $0 . " 5$ corresponds to a physically interesting resolving linear scale of $\sim 250 \mathrm{pc}$ at $100 \mathrm{Mpc}$.

Similarly, SKA1-MID will be a game-changer when it comes to providing a benchmark study for relating the CCSN rate to the SFR in both star-forming and normal spirals, and down to dwarf irregulars. With sensitivities of over an order of magnitude better than current instruments, and sub-arcsecond resolutions providing linear resolution scales of a few tens of pc within nearby galaxies, SKA1-MID will be well matched to spatially separate CCSNe from their surrounding diffuse emission, thus enabling a complete census.

With the 20 fold increase in angular resolution expected with the full SKA (resolution of about 10 mas at $1.67 \mathrm{GHz}$ (see also Paragi et al., 2015), it will be possible to locate individual core-collapse supernovae (or supernova remnants) within the nuclear regions of all local starburst galaxies, similar to detailed studies in e.g., Arp 220 and Arp 299 (see Fig. 4, taken from PérezTorres et al. 2009a, 2010), but with the potential of unveiling the much more numerous, fainter radio supernovae and supernova remnants. In turn, this will allow us to test scenarios of SN/CSMISM interaction, including estimates of the energy budgets in particles and magnetic fields, and determine the SNR luminosity vs. size relation for essentially all local (U)LIRGs. In addition, we will be able to extend the study described above to essentially all redshifts, as the 10 mas beam at $1.67 \mathrm{GHz}$ will yield spatial resolutions of $80 \mathrm{pc}$, or better, at all redshifts.

\section{Accretion sources in the nearest galaxies}

The sensitivity and frequency coverage of SKA1 will enable a complete census of accretion powered sources in the local Universe. Such sources encompass multiple orders of magnitude in mass and luminosity, from supermassive black-holes (SMBH) and low-luminosity AGN, through Sgr A*-like luminosities, to intermediate-mass black-holes and down to stellar mass black-hole 

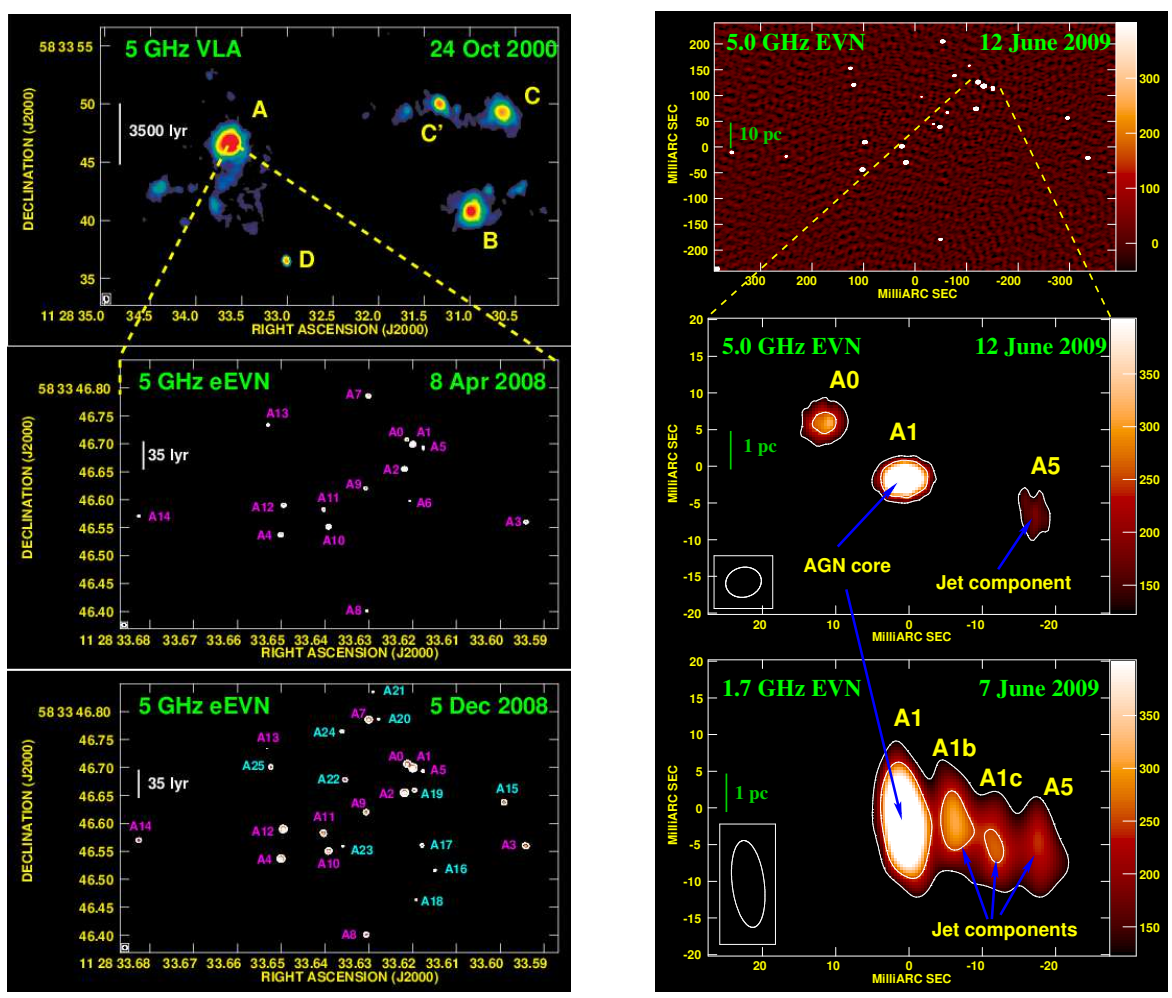

Figure 4: Top left: $5 \mathrm{GHz}$ VLA archival observations of Arp 299 on 24 October 2000, displaying the five brightest knots of radio emission in this merging galaxy. Middle and bottom left: Contour maps drawn at five times the r.m.s. noise of our $5 \mathrm{GHz}$ eEVN observations of the central 500 light years of the luminous infrared galaxy Arp 299-A on 8 April 2008 and 5 December 2008, revealing a large population of bright, compact, non-thermal emitting sources. To guide the reader's eye, we show in cyan the components detected only at the 5 December 2008 epoch. Top right: 5.0 GHz full EVN image of the central 150 parsec region of the luminous infrared galaxy Arp 299-A, displaying a large number of bright, compact, non-thermal emitting sources, mostly identified with young RSNe and SNRs. Middle and bottom right: Blow-ups of the inner 8 parsec of the nuclear region of Arp 299-A, as imaged with the full EVN at 1.7 and $5.0 \mathrm{GHz}$. The core-jet morphology, spectral index and luminosity of the A1-A5 region clearly revealed the location of the long-sought AGN in Arp 299A.

systems such as microquasars. The sensitivity of the SKA during a deep pointed survey will show accretion-dominated objects in a variety of environments within an individual galaxy, not constrained just to the nucleus.

\subsection{Accretion onto supermassive Black-holes}

Accretion onto SMBHs is one of the most significant energy sources in the universe, with the potential to clear star-forming gas from galactic bulges and even to regulate the growth of entire galaxies in galaxy clusters (e.g. di Matteo, Springel \& Hernquist, 2005). The mechanism for this feedback is mechanical, through jets and outflows powered by accretion. However, despite the importance of SMBH activity in regulating galaxy formation, comparatively little is known about SMBH activity towards low radiative luminosities. This is a significant gap in our understanding 
of feedback and the role of SMBHs in galaxy growth and evolution, since it is now known that mechanical jet power can be energetically more significant than supernova feedback, even at low AGN luminosities (Nagar, Falcke \& Wilson, 2005; Körding, Jester, \& Fender, 2008). The major difficulty in studying low luminosity AGN is precisely their low radiative output with respect to their surrounding host galaxy, especially since many LLAGN are embedded in nuclear SF regions (see Fig. 4; and work of Ho et al., 1997, and references therein). For these LLAGN, large amounts of mechanical energy are shed by massive stars into their surrounding medium which significantly increases its temperature. Thus those massive stars would hinder the accretion of material to the central black-hole, resulting in a less powerful AGN. The problem is compounded because at lowluminosities AGN become radiatively inefficient, and lose the strong optical and X-ray signatures commonly used to identify AGN activity. However, just because an AGN is radiatively inefficient does not make it mechanically inefficient, in fact at low luminosities the total power is almost certainly mechanically-dominated via the jet.

Radio imaging is also essential to unambiguously identify activity due to SMBH accretion. For example, even with more sensitive optical spectroscopy, it will be difficult to distinguish the weak optical emission from faint AGN against the contribution from nuclear SF. In contrast, a separation of AGN and SF components can be made with the moderate resolution ( $\left.\sim 0^{\prime \prime} 5\right)$ obtained by SKA1MID initially in the most local systems, coupled with the leverage of multiple frequency bands to distinguish flat-spectrum AGN cores. Finally, using radio data circumvents the problem of where to draw the dividing line between a galaxy being "active" or "normal". This is because one can not only estimate the jet power from core radio luminosities, but also measure the absolute mass accretion rate, which appears to be well-correlated with radio luminosity for the lowest luminosity objects, and where beaming effects do not play an important role, scaling as $\mathrm{L}_{\mathrm{R}} \propto \mathrm{M}_{\odot}^{1.4}$ (Körding, Jester, \& Fender, 2006). Thus we can classify all galaxies according to their SMBH accretion power, tying the lowest accretion "quiescent" SMBH to the LINERs and classical Seyferts.

\subsection{Accretion from intermediate to stellar mass black-holes}

Deep radio surveys of the local Universe with the SKA will prove critically important to our further understanding of high-energy accretion processes across a wide mass-function of compact objects. Bright off-nuclear X-ray point sources with luminosities in excess of $10^{39} \mathrm{erg} \mathrm{s}^{-1}$, known as Ultra-Luminous X-ray sources (ULXs), are among the most energetic mass-accretion processes in the local Universe (Roberts, 2007). Due to their defining characteristics, ULXs could be explained by stellar-mass black-holes with global accretion rates at or in excess of their Eddington limit or represent a population of intermediate mass black-holes (IMBH) with masses between $10^{2-3} \mathrm{M}_{\odot}$ (e.g., King, 2009). Some evidence for the latter has arisen through the discovery of radio emitting 'bubble nebulae', 100s of parsecs in diameter surrounding the X-ray sources (e.g., Pakull $\&$ Mirioni, 2002), and transient radio outbursts from a 'hyper-Luminous X-ray' source at a distance of $\sim 100 \mathrm{Mpc}$ (Webb et al., 2012). These may be photo-ionised by the central X-ray source (Pakull \& Mirioni, 2002; Kaaret, Ward \& Zezas, 2004), shock-heated through the interaction of outflows with the ambient medium (Pakull \& Mirioni, 2002, 2003) or both. As the bolometric radiative efficiency of these sources provides an unreliable measure of the black-hole mass, it is by studying these surrounding radio nebulae and jets that we could estimate the true kinetic power of these 
compact objects and gain insight to their mass-function (see more extensive chapter on this area by Wolter et al., 2015).

IMBHs and stellar-mass black-hole systems (e.g. microquasars) also provide a hitherto sparsely investigated population of transient or variable faint compact radio sources in galaxies and are expected in larger numbers in high SFR systems than are found in our own Galaxy (see Fender et al., 2015; Corbel et al., 2015, for more information on radio transients and the SKA). To date few dedicated radio searches have been performed of nearby star-forming galaxies and only a handful of candidate sources have been detected serendipitously (e.g., potenial microquasars in M82, Arp220 and Arp299; Muxlow et al., 2010; Joseph et al., 2011; Batejat et al., 2012; Bondi et al., 2012, respectively). Whilst the current radio instruments, such as JVLA and e-MERLIN, are only just starting to explore the brightest and nearest of this population. Via large-area and pointed SKA survey observations with sub-arcsecond angular resolution the entire population of accreting objects in galaxies across all mass scales and environments will be charactised. Searches for radio emission from star clusters and dwarf satellite galaxies around local galaxies will provide constraints on what fraction of each class of object shows evidence for containing black-holes of $\sim 1 / 1000$ of their total masses, in the same way that giant galaxies seem to do nearly universally. Several visits to the fields my be required to clarify the nature of the sources.

\section{Tracing the fuel for, and influence of SF and accretion: Molecular Gas tracers of kinematics and properties}

\subsection{OH masers and Circumnuclear starbursts}

Masers are the radio analogues of lasers, and occur when there is an excess of molecules in a higher energy state - a non-thermal or inverted population distribution. This gives a negative optical depth so that ambient or background radiation is amplified. Intrinsically compact both spatially and spectrally (extragalactic masers are detectable down to $\mu$ as scales in $<\mathrm{km} \mathrm{s}^{-1}$ channels), masers provide the best directly-mappable tracers of high-resolution kinematics. They demonstrate the presence of more compact molecular regions than any other $\mathrm{cm}$-wave tracer and increasingly sophisticated models (e.g. Gray 2012) place tight limits on the density-temperature parameter space required to pump the maser inversion. The most intense Milky Way masers are associated with individual star forming regions, and similar phenomena have been detected from Local Group galaxies, (water, methanol and hydroxl; Brunthaler et al., 2006; Sjouwerman et al., 2010; Argo et al., 2013, respectively). So called Mega- or kilo-masers can be more than a thousand time brighter and hence can be detected from within more distant galaxies (see Tarchi, 2012, for a recent review). Some of the potential science applications of the SKA for investigating nearby and Galactic masers is covered by Etoka et al. (2015) and Robishaw et al. (2015) in this proceedings.

OH masers, rest frequencies 1.6-1.7 GHz, have been found in over 100 (U)LIRGs out to $z=0.27$ (Darling \& Giovanelli, 2002; Willet, 2012). There is a strong relationship $L_{\mathrm{OH}} \propto\left(L_{\mathrm{IR}}\right)^{\alpha}$, explained by the rôle of radiation from dust in the maser pumping (Lockett \& Elizur, 2008). The corresponding OH IR lines at 35 and $53 \mu \mathrm{m}$ have recently been detected in both absorption $(53 \mu \mathrm{m})$ and emission by Herschel (González-Alfonso et al., 2014) providing important constraints on pumping schemes. Values of $0.5<\alpha<2$ have been reported, however these are sensitive to the angular resolution of the observations and the orientation of the emission region. Dust temperatures 
$45<T<200 \mathrm{~K}$ (optimally 80-140 K) and a number density $10^{9}<n<10^{12} \mathrm{~m}^{-3}$ (Darling 2007; Lockett \& Elizur 2008) are also required.

Images of extra-galactic $\mathrm{OH}$ masers have only been published for around a dozen galaxies further away than the Magellanic Clouds, out to $z \sim 0.045$. In approximate order of increasing distance, these are: M82 (Argo et al., 2010, 2013), NGC 1068 (Gallimore et al., 1996), III Zw35 (Parra et al., 2005), Arp 220 (Rovilos et al., 2003), Arp 299 (Polatidis \& Aalto, 2001), II Zw096 (Migenes et al., 2011), Mrk 273 (Yates et al., 2000), Mrk 213 (Richards et al., 2005), IRAS 172080014 (Momjian et al., 2006), IRAS 12032+1707, IRAS 1407+0525 (Pihlström et al., 2005) and IRAS 10173+0828 (Yu, 2005). All these galaxies show evidence for interactions or mergers and some masers are found at two or more sites hundreds of pc apart (e.g., in Arp220; Rovilos et al., 2003).

In most of the more distant objects, the brightest masers trace a rotating disc, typically a (few) hundred mas (a few hundred pc) in diameter, suggesting enclosed masses between $10^{6}-10^{9} \mathrm{M}_{\odot}$, possibly evidence for massive black-holes, but dense nuclear starburst regions, or both (Richards et al., 2005), cannot be ruled out without higher-resolution data (Klöckner, Baan \& Garrett, 2003). These Mega-masers are generally inside the extent of discs traced by HI. VLBI observations, and modelling of lower resolution data, show that the $\mathrm{OH}$ masers mostly emanate from clouds within a factor of four of $1 \mathrm{pc}$ in diameter (Lonsdale et al., 2003; Richards et al., 2005). The discs may also be warped or interact with jets, requiring sensitive high-resolution imaging for good models (e.g., Klöckner, Baan \& Garrett, 2003).

The $\mathrm{OH}$ ground state main lines, rest frequencies 1667.359 and $1665.402-\mathrm{MHz}$ are brightest, with intensity ratio $\geq 1.8$ (the value expected for unsaturated emission) and (in accordance with predictions), the highest brightness temperature masers have higher ratios (Lonsdale et al., 1998). The $\mathrm{OH}$ satellite lines at $1612-$ and $1720-\mathrm{MHz}$ are fainter and masers have only been detected in 6 objects (McBride, Heiles \& Elitzur, 2013). There are cases where 1720-MHz absorption accompanies 1612-MHz emission, requiring a different pumping mechanism even if mainline masers are also seen. A number of $\mathrm{OH}$ Mega-masers have line wings at up to $800 \mathrm{~km} \mathrm{~s}^{-1}$ from the systemic velocity (Baan, 1989), but neither these nor the satellite lines have ever been imaged.

\subsection{Other cm-wave extragalactic masers}

Bright 22-GHz extragalactic masers are best known thanks to their location in sub-pc tori around supermassive black-holes. Both Doppler and (with VLBI) proper motions were measured for NGC 4258 (Herrnstein et al., 1999; Green et al., 2015; Paragi et al., 2015). This gives the most precisely-known estimates of the enclosed black-hole mass and the distance, providing the disc kinematics are modelled accurately (Humphreys et al., 2013). The Hubble constant could thus be measured very accurately from a larger sample of such galaxies (e.g., the Mega-maser cosmology project Kuo et al., 2013). VLBI with the SKA will push this to far greater distances. A water maser at $z=2.6$ has already been detected at $6.1 \mathrm{GHz}$ (Impellizzeri et al., 2008) in a lensed galaxy. $\mathrm{H}_{2} \mathrm{O}$ masers are also found in interactions between jets and the ISM, (e.g. Kondratko, Greenhill \& Moran, 2003). Such galaxies do not usually have high SFRs and only a few nearby merger systems, e.g. NGC 1068, also host $\mathrm{OH}$ masers.

Excited $\mathrm{OH}$ lines occur in the rest frequency range $4-6.7 \mathrm{GHz}$; extragalactic thermal emission is known (Impellizzeri et al., 2006), but as yet these lines have not been observed as masers. 
Formaldehyde masers (rest frequency $4.8 \mathrm{GHz}$ ) are known from a handful of ULIRGs, all with $\mathrm{OH}$ Mega-masers (Araya et al., 2004). The inversion has the interesting property that maser amplification occurs at $n \geq 10^{11.6} \mathrm{~m}^{-3}$ and the excitation temperature exceeds the CMB temperature; at lower densities supercooling and strong absorption occurs (Mangum et al., 2013). Molecules, such as $\mathrm{OH}$ and $\mathrm{CH}$, with multiple masing transitions can be not only good thermometers, but also can be used as probes of any cosmological variation in fundamental constants.

\subsection{SKA phase 1 and SKA observations of Mega-maser molecules}

There are several complementary modes whereby SKA1-MID can locate and measure Megamaser properties. Spectral surveys would be very valuable in finding Mega-masers associated with galaxies out to the peak of the merger rate around redshift 1-2, helping to provide an estimate of the galaxy merger rate (Aharonian et al., 2013), and in identifying candidates for imaging. Searches are optimised by providing at least 3 samples across the line peak, i.e. a few tens $\mathrm{km} \mathrm{s}^{-1}$ channels, with angular resolution $\leq 1 \mathrm{arcsec}$, not greatly exceeding the size of the emission area. Nonetheless, piggyback observations at coarser resolution would be worthwhile. Selection criteria are described based on IR properties (Darling \& Giovanelli, 2002) including deep silicate $9.8 \mu \mathrm{m}$ absorption (Willett et al., 2011). The 18 sources detected at $0.1<z<0.27$ have $\mathrm{OH}$ peaks of $1.8-40 \mathrm{mJy}$ and line widths (FWHM) of 50-600 $\mathrm{km} \mathrm{s}^{-1}$, with some of the most distant being among the brightest. This suggests that a few percent of similarly selected candidates would have a flux density $\sim 0.6$ mJy at $z=1$ and the brightest might reach $\sim 0.2 \mathrm{mJy}$ at $z=2$. Assuming that a sensitivity of $0.13 \mathrm{mJy}$ can be reached in $20 \mathrm{~km} \mathrm{~s}^{-1}$ channels in $\leq 1 \mathrm{hr}$ (depending on Dec.) at 1 arcsec resolution, this would give a $5 \sigma$ detection for $0.6 \mathrm{mJy}$ masers in $1-2 \mathrm{hr}$, at modest redshifts around $1.6 \mathrm{GHz}$. At $555 \mathrm{MHz}, z \sim 2$, the lower sensitivity requires $\sim 24 \mathrm{hr}$ to detect $0.2 \mathrm{mJy}$ lines. McKean \& Roy (2009) describe a strategy using APERTIF or ASKAP for a blind search which would detect $\sim 1$ mJy masers $(5 \sigma)$ at the highest accessible redshift of $z=1.39$, but in several hours (per tuning) and $150 \mathrm{~km} \mathrm{~s}^{-1}$ resolution.

The known satellite lines are 1-10\% of the main line peaks in Mega-maser galaxies, but are often brighter in Milky-Way-like star-forming regions. McBride, Heiles \& Elitzur (2013) detected 5 out of 77 sources at $z<0.05$, at a few mJy. The 1-hr sensitivity of SKA will go at least twice as deep. Detection of multiple maser lines places tighter constraints on the parameter space of temperature-density-velocity coherence. Such a survey may also produce a large enough sample of satellite maser lines to help identify the pumping mechanism responsible for conjugate behavior, especially for any bright enough to image.

The enclosed mass density derived from resolved maser PV diagrams can be compared with predictions from thermal tracers of high-density, warm gas, for example HCN, which has excitational properties correlated with OH Mega-masers (Darling, 2007; Kandalyan \& Al-Zyout, 2010, and see Section 4.5) and with $\mathrm{HC}_{3} \mathrm{~N}$ (Lindberg et al., 2011). The position of maser components can be measured with a precision (for reasonable uv coverage) of $0.5 \times$ (beamsize/signal-to-noise ratio), so, at 250 mas resolution, a signal-to-noise of 5 across a few hundred $\mathrm{km} \mathrm{s}^{-1}$, sampled by 5 or more channels, would resolve a $\sim 150 \mathrm{pc}$ radius edge-on disc at $z \sim 0.2$. The 2 -hr sensitivity at this resolution in $30 \mathrm{~km} \mathrm{~s}^{-1}$ channels is $\sim 0.5 \mathrm{mJy}$, allowing emission above $2.5 \mathrm{mJy}$ to be resolved sufficiently. This would typically correspond to a $10 \mathrm{mJy}$ spectral peak. 
Selected objects should be imaged for 12-24 hr, to allow higher sensitivity or higher resolution. This will allow detection of multiple components per channel from discs tilted enough to be spatially resolved, and define the angle of inclination. The $1665-\mathrm{MHz}$ line is typically fainter and lacks the compact hot-spots seen in the 1667-MHz line; imaging this avoids blending ambiguities as well as providing material for maser modelling. Higher sensitivity will provide the first imaging of the satellite lines, providing tighter constrains on physical conditions. It will also reveal the origin of the high-velocity mainline emission, which could be fast-rotating regions in the inner disc, or material entrained by jets (Klöckner, Baan \& Garrett, 2003), since at least some of the host galaxies contain weak AGN, although the fraction has been variously estimated at 45\% (Baan et al., 1998) or 10-25\% (Willett et al., 2011). Another possibility is a molecular outflow as suggested by the velocity dispersion within the III Zw35 disc (Parra et al., 2005) since the superwind from NGC 253 has recently been shown by ALMA to be laden with molecules as well as the better-known light, ionised component (Walter et al., 2013).

Searches and rapid imaging of extragalactic masers requires a channel width of a few tens of $\mathrm{km} \mathrm{s}^{-1}$ at most, since maser lines tend to be narrow and diluted at too-coarse a resolution. Spectra can be decomposed into a mixture of broad features and few $\mathrm{km} \mathrm{s}^{-1}$ details, and a resolution of $\sim 1 \mathrm{~km} \mathrm{~s}^{-1}$ is needed to measure Zeeman splitting (Robishaw et al., 2015), or even finer for the measurement of fundamental constants. Similarly, comparison of single dish, WSRT, MERLIN and EVN spectra (Klöckner, 2004) shows that the brightest peaks are often almost as bright at $~ 30$ mas resolution as at a resolution of a few arcsec or more, but the weaker emission is partly or completely resolved out on scales of a few hundred mas or less. The SKA will provide the first opportunity to sample emission on MERLIN to WSRT scales simultaneously (and eventually on VLBI scales). Flexible data reduction is needed, with weighting-up of short baselines for maximum sensitivity at high spectral resolution, and channel averaging to allow high spatial resolution making full use of long baselines.

Finally, another motive for characterising $\mathrm{OH}$ masers is to avoid contamination of $\mathrm{HI}$ surveys; the maser lines can be distinguished at higher spectral resolution or by anomalous redshift and excess brightness with respect to other lines and continuum/optical properties (Haynes et al., 2011).

\subsection{Summary of Mega-maser requirements}

The basic requirement is the frequency range. Ground-state $\mathrm{OH}$ masers at $0<z<2$ need frequencies from about 1721 down to $530 \mathrm{MHz}$. Searches are optimized at a velocity resolution of ten or a few tens of $\mathrm{km} \mathrm{s}^{-1}$. Follow-up of brighter objects at resolution down to $\leq 1 \mathrm{~km} \mathrm{~s}^{-1}$ in full polarization is desirable, with a coverage of a few thousand $\mathrm{km} \mathrm{s}^{-1}$ and angular resolution as fine as possible (ideally 100 mas or less). However, it will not be possible to achieve many detections simultaneously at full spectral and spatial resolution. To achieve this the use of different weighting may be required to produce the highest spectral or spatial resolution images.

The (to date) rarer extragalactic masers such as $\mathrm{CH}$, formaldehyde, methanol and excited $\mathrm{OH}$ require frequencies up to just above $6 \mathrm{GHz}$ (potentially higher, since Galactic $\mathrm{OH}$ and methanol are known up to $13 \mathrm{GHz}$ ). In fact, any expansion of frequency range will allow detection of masers at some redshift, notably for the $22-\mathrm{GHz}$ water masers. In the more advanced stages of the SKA, VLBI at up to $22 \mathrm{GHz}$ is essential for the Megamaser Cosmology Project. 


\subsection{Molecular line and other tracers}

There is now mounting evidence that cold, neutral/molecular outflows are extremely important carriers of momentum and mass within galaxies. We are beginning to see that the properties and mass balance atomic/molecular/ionised change along individual outflows as well as among outflows. In this area HI absorption studies are absolutely fundamental, however observations of $\mathrm{OH}$ maser emission in dense outflows in ULIRGs also have a critical role and radio recombination lines (RRLs) also potential can play an important part in nearby sources (see section 4 and Morganti et al., 2015; Oonk et al., 2015, for HI absorption and RRL opportunities with the SKA). Very recently it has also become clear that AGN driven outflows may also have extremely interesting molecular properties (linked to their evolution) that can be studied in exquisite detail with the SKA. The ISM properties of galaxies throughout the Universe can be investigated via HI and radio recombination studies which probe the atomic and ionized medium and are a key complement to the molecular cloud structure/mass/dynamics probed by mm-telescopes such as ALMA. This area is of fundamental importance to the understanding of cloud and SF processes in galaxies both near and far.

In this area the sensitivity and frequency coverage of the SKA, even in phase-1, provides a unique opportunity to study these processes via centimetric molecular lines that have hitherto been ill explored (preliminary JVLA and Arceibo studies have been made on selected sources e.g., Rickert, 2011; Salter et al., 2008). A number of molecular tracers, which include several prebiotic molecules, are available within the proposed SKA-1 bands (see Table 1) and will be observable as kinematic and ISM tracers within local galaxies.

For example the SKA's capabilities provide the possibility of studying the properties of the extreme nuclear regions of Compact Obscured Nuclei (CONs) - and dust obscured galaxies (DOGs). Here, activity may be buried behind $\mathrm{N}_{\mathrm{H}}>1 \times 10^{25} \mathrm{~cm}^{-2}$ of gas which means that these Compton thick, high pressure regions are the realm of molecules (not even X-rays will penetrate to help fingerprint the activity). Molecular emission can probe behind the optically thick dust veil to reveal temperature gradients and field strengths at SKA spatial resolution. Thus these studies will trace the impact and structure of the deeply buried activity in a unique way. The properties of these galactic nuclei may only be accessible with molecules.

Why SKA and not ALMA? Many of these line species are accessible at mm and submm wavelengths (and studies are underway with ALMA). However, some of the heavier molecules have their primary transitions in the SKA bands, plus critically at submm wavelengths the line confusion and line blending is so severe that using these lines sometimes becomes extremely difficult. Pilot studies on nearby galaxies are underway on the JVLA at 6,9 and $20 \mathrm{GHz}$, however these will remain sensitivity limited with current facilities. The sensitivity and frequency range covered by the SKA make this it an ideal instrument to open up this new field. Furthermore initial modelling work predicts that several of these molecules will have maser transitions at the very lowest frequencies (e.g. $0.3 \mathrm{GHz}$ ) rendering them as potentially extremely important probes of circumnuclear extreme regions around AGNs.

\section{Conclusions}

The major endeavor that is the SKA will create a general user facility which will produce 
Table 1: Selection of molecular line tracers between 1-10 GHz and within the bands of SKA-1. Lines of the $\mathrm{OH}$ radical and various other species are also of key importance but for brevity not included below.

\begin{tabular}{lccc}
\hline Line & Rest freq (MHz) & SKA-1 MID band & SKA-1-SUR band \\
\hline $\mathrm{HCN} v_{2}=1, \Delta J=0, J=2$ & 1346.765 & 2 & 2 \\
$\mathrm{HCN} v_{2}=1, \Delta J=0, J=4$ & 4488.4718 & 4 & - \\
$\mathrm{H}_{2} \mathrm{CO}$ & 4829 & $4 / 5$ & - \\
$\mathrm{N}_{2} \mathrm{H}+$ & 5009.8278 & $4 / 5$ & - \\
$\mathrm{H}_{2} \mathrm{CNH} 1_{10}-1_{11}, \Delta F=0, \pm 1$ & 5289.813 & 5 & - \\
$\mathrm{HCO}+$ & 6350.908 & 5 & - \\
$\mathrm{HNC}$ & 6484.497 & 5 & - \\
$\mathrm{CH}_{3} \mathrm{OH} 5_{1}-6_{0} A^{+}$ & 6668.5192 & 5 & - \\
$\mathrm{HCN} v_{2}=1, \Delta J=0, J=5$ & 6731.9098 & 5 & - \\
$\mathrm{HCO}+$ & 8890.452 & 5 & - \\
$\mathrm{HCN} v_{2}=1, \Delta J=0, J=6$ & 9423.3338 & 5 & - \\
$\mathrm{HNC}$ & 9724.644 & 5 & - \\
\hline
\end{tabular}

transformational science across a wide range of astrophysics for many decades to come. For studies of the galaxy population within the local Universe these broad, general user capabilities, will enable an extremely wide range of science covering many areas of astrophysics, and will form the bridge between the detailed studies of objects in our own Galaxy and the distant high-redshift Universe. Deep and moderate-resolution (few arcsec) continuum and spectral line surveys of large numbers of nearby galaxies will be provided by projected SKA1 "all-sky" surveys at frequencies of $\sim 1$ $2 \mathrm{GHz}$. Such large area surveys will essentially provide a full radio atlas of the local Universe and allow detailed studies of the non-thermal radio component of galaxies. However, multiple, pointed observations covering a wide range of frequency bands, in particular higher band SKA1MID (band 5), will be required to characterise the non-thermal components of local galaxies. Even with modest capability reductions, SKA1 will make significant advances in this field. This will, for the first time, allow the determination of SF within galaxies covering the full range of type and environments which will be critical to our understanding of galaxy evolution and SF through cosmic time.

Via higher frequency bands in SKA1 and later baseline extensions during the full SKA high angular resolution ( $>0$. "5 to mas) continuum and spectral line (maser and absorption studies) of nearby galaxies will allow the physical composition of these galaxies to be decomposed. Even with the modest resolution of SKA1, this will result in the detection and study of many thousands of individual SF and accretion-related components such as SNe, SNR, HII and their like. Via such observations the SKA will not only be able to study the physics of SF and extreme physics in accretion dominated sources on an individual basis in nearby galaxies, but also allow statistical properties of these sources to be investigated, how they interact with the ISM and how they affect galaxy evolution. 


\section{References}

Aharonian, F., Arshakian, T. G., Allen, B., et al. 2013, Pathway to the Square Kilometre Array The German White Paper, Ed. Köckner et al.

Alberdi, A., Colina, L., Torrelles, J. M., et al. 2006, ApJ, 638, 938

Araya, E., Hofner, P., Kurtz, S., et al., 2004, ApJ, 154, 541

Argo, M. K., Pedlar, A., Beswick. R. J., et al, 2010, MNRAS, 402, 2703

Argo, M. K., Beswick, R. J., Muxlow, T. W. B., et al., 2013, POS (11th EVN Symposium) 05 Appleton, P. N., Fadda, D. T., Marleau, F. R. et al. 2004, ApJS, 154, 147

Baan, W. A., 1989, ApJ, 346, 680

Baan, W. A. Salzer, J. J., LeWinter, R. D, 1998, ApJ, 509, 633

Batejat, F., Conway, J. E., Rushton, A., et al. 2012, A\&A, 542, 24

Beck, R., \& Krause, M. 2005, Astronomische Nachrichten, 326, 414

Beck, R. et al. "Structure, dynamical impact and origin of magnetic fields in nearby galaxies in the SKA era", in proceedings of "Advancing Astrophysics with the Square Kilometre Array", 2015, POS (AASKA14) 094

Beswick, R. J., Muxlow, T. W. B. Thrall, H., et al 2008, MNRAS, 385, 1143

Bell, E. F. 2003, ApJ, 586, 794

de Blok, E. et al. "The SKA view of the Neutral Interstellar Medium in Galaxies", in proceedings of "Advancing Astrophysics with the Square Kilometre Array", 2015, PoS (AASKA14) 129

Bondi. M., Perez-Torres, M. P., Dallacasa, D., et al. 2005, MNRAS, 361, 748

Bondi, M., Perez-Torres, M. A., Herrero-Illana, R, et al. 2012, A\&A, 539, 134

Braun, R., Oosterloo, T. A., Morganti, R., et al. 2007, A\&A, 461, 455

Brunthaler, A., Henkel, C, de Blok et al., 2006, A\&A, 457, 109

Colina, L., Alberdi, A., Torrelles, J. M., et al. 2001, ApJ, 553, L19

Condon, J. J. 1992, ARA\&A, 30, 575

Condon, J. J., Cotton, W. D., \& Broderick, J. J. 2002, AJ, 124, 675

Corbel, S. et al. "Incoherent transient radio emission from stellar-mass compact objects in the SKA era", in proceedings of "Advancing Astrophysics with the Square Kilometre Array", 2015, POS (AASKA14) 053

Darling, J. \& Giovanelli, R., 2002, AJ, 124, 100

Darling, J., 2007, ApJ, 669, L9

Dumas, G., Schinnerer, E., Tabatabaei, F. S, et al, 2011, AJ, 141, 41

Etoka, S. et al. "OH masers in the Milky Way and Local group galaxies in the SKA era", in proceedings of "Advancing Astrophysics with the Square Kilometre Array", 2015, POS (AASKA14) 125

Fender, R. et al. "The Transient Universe with the Square Kilometre Array ", in proceedings of "Advancing Astrophysics with the Square Kilometre Array", 2015, PoS (AASKA14) 051

Fenech, D. M., Muxlow, T. W. B., Beswick, R. J., et al. 2008, MNRAS, 391, 1384

Fenech, D. M., Beswick, R. J., Muxlow, T. W. B., et al. 2010, MNRAS, 408, 607

Gallimore, J. F., Baum, S. A., O’Dea C. P., et al., 1996, ApJ, 462, 740

Garrett, M. A. 2002, A\&A, 384, 19

Gebhardt, K., Rich, R. M., \& Ho, L. C. 2005, ApJ, 634, 1093 
Gendre, M. A., Fenech, D. M., Beswick, R. J., et al. 2013, MNRAS, 431, 1107

González-Alfonso, E., Fischer, J., Garciá-Carpio J., et al., 2014, A\&A, 561, A27

Gray, M., Maser Sources in Astrophysics, CUP 2012

Green, J., et al. "Maser Astrometry with VLBI and the SKA", in proceedings of "Advancing Astrophysics with the Square Kilometre Array", 2015, PoS (AASKA14) 119

Haynes, M. P., Giovanelli, R., Martin, A. M., et al., 2011, AJ, 142, 170

Heald, G. et al. "Magnetic field tomography in nearby galaxies with the Square Kilometre Array", in proceedings of "Advancing Astrophysics with the Square Kilometre Array", 2015, POS (AASKA14) 106

Heesen, V., Rau, U., Rupen, M. P., et al. 2011, ApJ, 739, L23

Heesen, V., Brinks, E., Leroy, A. K. et al. 2014, AJ, 147, 103

Helou, G., Soifer, B. T. \& Rowan-Robinson, M. 1985, ApJ, 298, L7

Herrnstein, J. R., Moran, J. M., Greenhill, L. J., et al., 1999, Nature., 400, 539

Ho, L. C., Filippenko, A. V., Sargent, W. L. W. 1997, ApJ, 487, 568

Humphreys, E. M. L., Reid, M. J., Moran, J. M., et al., 2013, ApJ, 755, 13

Impellizzeri, C. M. V., Roy, A. L. \& Henkel, C. 2006, PoS (8th EVN Symposium)

Impellizzeri, C. M. V., McKean, J. P., Castangia, P., et al., 2008, Nature., 456, 927

de Jong, T., Klein, U., Wielebinski, R., \& Wunderlich, E. 1985, A\&A, 147, L6

Joseph, T. D., Maccarone, T. J., \& Fender, R. P. 2011, MNRAS, 415, 59

Kaaret, P., Ward, M. J., \& Zezas, A., 2004 MNRAS, 351, 83

Kandalyan, R. A. \& Al-Zyout, M. M.,2010 Astrophysics, 53, 475

Kankare, E., Mattila, S., Ryder, S., et al., 2008, ApJ, 687, 97

Kennicutt, R. C., Jr. 1998, ApJ, 498, 541

King, A. R., 2009, MNRAS, 393, 41

Kitchener, B., Brinks, E., Heesen, V., et al. 2014, AJ, (submitted)

Klöckner, H.-R., Baan, W. A. \& Garrett, M. A. 2003, Nature, 421, 821

Klöckner, H.-R., 2004, PhD Thesis, University of Groningen

Kondratko, P. T., Greenhil L. J. \& Moran, J. M., 2003, ApJ, 618, 618

Körding, E. G., Colbert, E. \& Falcke, H. 2005, A\&A, 436, 427

Körding, E. G., Jester, S. \& Fender, R. 2006, MNRAS, 372, 1366

Körding, E. G., Jester, S. \& Fender, R. 2008, MNRAS, 383, 277

Kuo, C. Y., Braatz, J. A., Reid, M. J., et al., 2013, ApJ, 767, 155

Lacki, B. C., \& Thompson, T. A. 2010, ApJ, 717, 196

Lacki, B. C., Thompson, T. A., \& Quataert, E. 2010, ApJ, 717, 1

Li, Y., Calzetti, D., Kennicutt, R. C., et al. 2010, ApJ, 725, 677

Lindberg, J. E., Aalto, S., Costagliola, F., et al., 2011, A\&A, 527, A150

Lisenfeld, U., Völk, H. J., \& Xu, C. 1996, A\&A, 314, 745

Lockett, P. \& Elizur, M., 2008, ApJ, 677, 985

Lonsdale, C. J., Lonsdale, C. J., Smith, H. E., et al., 1998 ApJ, 493, L13

Lonsdale, C. J., Lonsdale, C. J., Smith, H. E., et al., 2003, ApJ, 592, 804

Lonsdale, C. J., Diamond, P. J., Thrall, H., et al. 2006, ApJ, 647, L185

Mancuso, C. et al. "Radio Observations of Star-forming Galaxies in the SKA era", in proceedings of "Advancing Astrophysics with the Square Kilometre Array", 2015, POS (AASKA14) 082 
Mangum, J. G., Darling, J., Henkel, C. et al., 2013, AJ, 766, 108

di Matteo, T., Springel, V. \& Herquits, L. 2005, Nature, 433, 605

Mattila, S., Dahlen, T., Efstathiou, A., et al., 2012, ApJ, 756, 111

McBride, J., Heiles, C., Elitzur, M. 2013, ApJ, 774, 35

McKean, J. \&Roy, A. L., 2009, Panoramic Radio Astronomy, PoS, 89, 60

Migenes, V., Coziol, R., Cooprider, K., et al., 2011, MNRAS, 416, 12

Momjian, E., Romney, J. D., Carilli, C. L., et al., 2006, ApJ, 653, 1172

Morganti, R. et al. "Cool Outflows and HI absorbers with SKA", in proceedings of "Advancing Astrophysics with the Square Kilometre Array", 2015, PoS (AA.SKA14) 134

Murphy, E. J., Braun, R., Helou, G., et al. 2006, ApJ, 638, 157

Murphy, E. J., Helou, G., Kenney, J. D. P., et al. 2008, ApJ, 678, 828

Murphy, E. J. 2009, ApJ, 706, 482

Murphy, E. J., Condon, J. J., Schinnerer, E. et al. 2011, ApJ, 737, 67

Murphy, E. J., Bremseth, J., Mason, B. S., et al. 2012, ApJ, 761, 97

Murphy, E. J., Sargent, M., T, Beswick, R. J., et al. "The Astrophysics of Star Formation Across Cosmic Time at $>10 \mathrm{GHz}$ with the Square Kilometer Array", in proceedings of "Advancing Astrophysics with the Square Kilometre Array", 2015, PoS (AASKA14) 085

Muxlow, T. W. B., Beswick, R. J., Garrington, S. T., et al. 2010, MNRAS, 404, 109

Nagar, N. M., Falcke, H. \& Wilson, A. S. 2005, A\&A, 435, 521

Neff, S. G., Ulvestad J. S., Teng, S. H. 2004, ApJ, 611, 186

Niklas, S., \& Beck, R. 1997, A\&A, 320, 54

Norris, R. P., Afonso, J., Bacon, R. D., et al 2013, PASA, 30, 20

Oonk, R., et al. "The Physics of the Cold neutral Medium: Low-frequency Radio recombination Lines with the SKA", in proceedings of "Advancing Astrophysics with the Square Kilometre Array", 2015, POS (AASKA14) 139

Pakull, M. W., \& Mirioni, L., 2002, in proceedings of "New Visions of the X-ray Universe in the XMM-Newton and Chandra Era, astro-ph/0202488

Pakull, M. W., \& Mirioni, L., 2003, Rev. Mexicana Astron. Astrofis.15, 197

Paladino, R. et al. "Synergies between SKA and ALMA: observations of Nearby Galaxies", in proceedings of "Advancing Astrophysics with the Square Kilometre Array", 2015, POS (AASKA14) 156

Paragi, Z., Godfrey, L., Reynolds, C., et al. "Very Long baseline Interferometry with the SKA", in proceedings of "Advancing Astrophysics with the Square Kilometre Array", 2015, POS (AASKA14) 143

Parra, R., Conway, J. E., Elitzur, M. et al., 2005, A\&A, 443, 383

Parra, R., Conway, J. E., Diamond, P. J., et al. 2007, ApJ, 659, 314

Pedlar, A 2001, IAU Symp., 205, 366

Peel, M. W., Dickinson, C., Davies, R. D., et al. 2011, MNRAS, 416, 99

Pérez-Torres, M. A., Mattila, S., Alberdi, A., et al. 2007, ApJ, 671, L21

Pérez-Torres, M. A., Romero, C., Alberdi, A., et al. 2008, CBET, 1392, 2P

Pérez-Torres, M. A., Romero-Cañizales, C., Alberdi, A., et al 2009a, A\&A, 507, L17

Pérez-Torres, M. A., Alberdi, A., Colina, L., et al 2009b MNRAS399, 1641

Pérez-Torres, M. A., Alberdi, A., Romero-Cañizales, C., et al 2010, A\&A, 519, L5 
Pérez-Torres, M. et al. "Radio emission from young CCSNe and Type Ia $\mathrm{SNe}$ with the SKA", in proceedings of "Advancing Astrophysics with the Square Kilometre Array", 2015, POS (AASKA14) 060

Pihlström Y. M., Baan, W. A., Darling, J., et al. 2005, ApJ, 618, 705

Planck Collaboration, 2013, 2013arXiv1303.5076P

Polatidis, A. G. \& Aalto, S., 2001, IAU Symp. 205, 2011

Prandoni, I. \& Seymour, N, "Revealing the Physics and Evolution of Galaxies and Galaxy Clusters with SKA Continuum Surveys", in proceedings of "Advancing Astrophysics with the Square Kilometre Array", 2015, POS (AASKA14) 067

Price, R., \& Duric, N. 1992, ApJ, 401, 81

Richards, A. M. S., Knapen, J. H., Yates, J. A. et al., 2005, MNRAS, 364, 353

Rickert, M. 2011 MSc thesis, DePaul University, Chicago

Roberts, T. P., 2007, Ap\&SS, 311, 203

Robishaw, T. et al. "Measuring Magnetic Fields Near and Far with the SKA via the Zeeman Effect", in proceedings of "Advancing Astrophysics with the Square Kilometre Array", 2015, POS (AASKA14) 110

Rovilos, E., Diamond, P. J., Lonsdale, C. J., et al., 2003, MNRAS, 342, 373

Rovilos, E., Diamond, P. J., Lonsdale, C. J., et al. 2005, MNRAS, 359, 827

Salter, C. J., Ghosh, T., Catinella, B. et al. 2008, AJ, 136, 389

Schleicher, D. R. G., \& Beck, R. 2013, A\&A, 556, A142

Sjouwerman, L. O., Murray, C. E., Pihlström, Y. M., et al. 2010 ApJ, 724, 158

Smith, H. E., Lonsdale, C. J., Lonsdale, C. J., et al. 1998,ApJ, 493, L17

Tabatabaei, F. S,. Schinnerer, E. Murphy, E. J., et al. 2013, A\&A, 552, 19

Tarchi, A., 2012, IAU Symposium 287, CUP, 323

Trudolyubov \& Priedhorsky 2004 ApJ, 616, 821

Ulvestad, Greene \& Ho 2007, ApJ, 616, 151

Völk, H. J. 1989, A\&A, 218, 67

Walter, F., Bolatto, A. D., Leroy, A. K., et al. 2013, AAS, 22122105

Wehner, E. H., \& Harris, W. E. 2006, ApJ, 644, L17

Webb, N., Cseh, D., Lenc, E., et al. 2012, Science, 337, 554

Weiler, K. W., Panagia, N., Montes, M .J., et al. 2002, ARA\&A, 40, 387

Willett, K. W., Darling, J., Spoon, H. W. W., et al., 2011, AJ, 73056

Willett, K. W., 2012, IAU Symposium 287, CUP, 345

Wolter, A. et al "Radio investigations of Ultra-luminous X-ray (ULX) sources in the SKA Era", in proceedings of "Advancing Astrophysics with the Square Kilometre Array", 2015, POS (AASKA14) 091

Yates, J. A., Richards, A. M. S., Wright, M. M. et al., 2000, MNRAS, 317, 28

Yu, Z.-Y., 2005, Chin. JAA, 5, 2, 159

Yun, M. S., Reddy, N. A., \& Condon, J. J. 2001, ApJ, 554, 803 